\title{
Sobrepeso/Obesidade em Adolescentes \\ Escolares da Cidade de João Pessoa - PB: \\ Prevalência e Associação com Fatores \\ Demográficos e Socioeconômicos
}

\section{Overweight/Obesity in Adolescent Students From the City of João Pessoa, PB, Brazil: Prevalence and Association with Demographic and Socioeconomic Factors}

José Cazuza de Farias Júnior1,2,3

Kelly Samara da Silva

1. UFSC/CDS/PPGEF - Florianópolis

- SC, Brasil

2. UFPB/CCS/DEF - João Pessoa

- PB, Brasil

3. Bolsista CAPES

\section{Endereço para correspondência: Rua: Capitão Romualdo de Barros, 997 - Apto. 303 - Bl. B1, Carvoeira Florianópolis, SC - CEP: 88040-600 Fone: (48) 3209-7713 \\ Email: jcazuzaj@hotmail.com}

Submetido em 16/01/2007 Versão final recebida em 17/05/2007 Aceito em 23/10/2007

\begin{abstract}
RESUMO
Objetivos: O sobrepeso/obesidade na infância e adolescência tem sido considerado como um dos maiores problemas de saúde pública na atualidade, em função do rápido crescimento na sua prevalência e da associação com diversos problemas de saúde. Nesse sentido, o objetivo deste estudo foi determinar a prevalência de sobrepeso/obesidade em adolescentes escolares do ensino médio da cidade de João Pessoa - PB, e analisar sua associação com fatores demográficos e socioeconômicos. Métodos: Participaram do estudo 2.402 escolares do ensino médio (escolas públicas e privadas), de 14 a 18 anos (16,5 $\pm 0,2)$. Além de informações demográficas (sexo e idade) e socioeconômicas (classe econômica, escolaridade dos pais), os escolares referiram o peso corporal $(\mathrm{kg})$ e a estatura $(\mathrm{cm})$. O sobrepeso/obesidade foi determinado pelo índice de massa corporal $(\mathrm{IMC}=$ peso[kg]/estatura[m²]) a partir dos critérios propostos pelo International Obesity Task Force. Resultados: A prevalência de sobrepeso/obesidade foi 10\%, mostrando-se mais elevada nos rapazes do que nas moças $(13,5 \%$ vs $7,4 \%$, razão de prevalência $[R P]=1,82 ;$ IC95\%=1,42-2,32) e dissociada da idade em ambos os sexos. Os resultados da análise multivariável evidenciaram maior probabilidade de sobrepeso/obesidade nos rapazes que pertenciam às classes econômicas mais elevadas ( $R P=2,75$; IC $95 \%=1,56-4,82$ ). Por outro lado, a probabilidade de sobrepeso/obesidade foi menor nas moças cujos pais apresentavam maior nível de escolaridade $(\mathrm{RP}=0,42 ; \mathrm{IC} 95 \%=0,21-0,87)$. Conclusões: A prevalência de sobrepeso/obesidade nos adolescentes escolares de João Pessoa - PB foi similar a que tem sido observado em estudos nacionais. Os rapazes mais ricos e as moças pobres foram os subgrupos de maior chance de apresentar sobrepeso/obesidade. Nesse sentido, no desenvolvimento de ações intervencionistas, deve-se levar em consideração as diferenças na freqüência de sobrepeso/obesidade em função dos estratos demográficos e socioeconômicos.
\end{abstract}

Palavras-chave: sobrepeso, obesidade, adolescente, fatores demográficos, fatores socioeconômicos.

\section{ABSTRACT}

Objectives: Overweight/obesity in childhood and adolescence has been described as one of the greatest public health problems of current times, in recognition of the rapid growth in its prevalence and its relationship with many different health problems. In this idea, the objective of this study was to determine the prevalence of overweight/obesity in high school adolescents from the city of João Pessoa, PB, and to analyze its relationship with demographic and socio-economic factors. Methods: The study sample consisted of 2,402 high school students (public and private schools), aged 14 to 18 years (16.5 \pm 0.2$)$. In addition to demographic details (age and sex) and socio-economic data (economic class, parents educational background), the students reported their body weight $(\mathrm{kg})$ and height $(\mathrm{cm})$. Overweight/obesity was determined based on body mass index (BMl= weight $[\mathrm{kg}] /$ stature $\left.\left[\mathrm{m}^{2}\right]\right)$ according to criteria proposed by the International Obesity Task Force. Results: The prevalence of overweight/obesity was 10\% and was more common among boys than girls $(13.5 \%$ vs. $7.4 \%$, prevalence ratio $[P R]=1.82 ; 95 \% \mathrm{Cl}=1.42-2.32)$ and had no association with age in either sex. Results of a multivariate analysis found evidence of greater probability of overweight/obesity among boys from higher economic classes ( $\mathrm{PR}=2.75 ; 95 \% \mathrm{Cl}=1.56-4.82)$. Conversely, among the girls the probability of overweight/obesity was lower for girls whose parents had a higher education background level ( $P R=0.42$; $95 \% \mathrm{Cl}=0.21-0.87$ ). Conclusions: The prevalence of overweight/obesity among adolescent students in João Pessoa, PB was similar to what has been observed in other Brazilian studies. The richer boys and poorer girls were the subsets with the greatest chance of exhibiting overweight/obesity. Within this context, intervention actions should take into consideration the different prevalence rates of overweight/obesity in different demographic and socio-economic strata.

Keywords: overweight, obesity, adolescent, socio-economic factors, demographic factors. 


\section{INTRODUÇÃO}

O sobrepeso/obesidade na infância e adolescência, assim como nos adultos, tem sido considerado como um dos maiores problemas de saúde pública da atualidade, tanto em países desenvolvidos como naqueles considerados de economia emergente, em função da estreita relação com diversos problemas de saúde, dos gastos crescentes com o tratamento dos agravos decorrentes, e do rápido crescimento na sua prevalência.

Na adolescência, o sobrepeso/obesidade tem sido associado à presença de fatores de risco para doença arterial coronariana ${ }^{(1)}$, níveis elevados de ansiedade e depressão, redução nos níveis de auto-estima e no desempenho escolar ${ }^{(2)}$. Além disso, representa um forte preditor de obesidade, de doenças cardiovasculares, e mortalidade precoce na fase adulta da vida(3).

O rápido crescimento na prevalência de sobrepeso/obesidade na população jovem tem sido amplamente documentado. Levantamentos populacionais indicam que, nas últimas três décadas, a prevalência de sobrepeso/obesidade nos adolescentes aumentou, cerca de duas a três vezes, em países desenvolvidos ${ }^{(4-6)}$ e em desenvolvimento, inclusive no $\operatorname{Brasi}\left({ }^{(4,7)}\right.$.

Dados brasileiros com relação à prevalência de sobrepeso/obesidade em amostras representativas da população jovem ainda são relativamente escassos, sobretudo que tenham focado a influência de fatores socioeconômicos na sua distribuição. Essa escassez torna-se ainda mais evidente quando se levam em consideração estudos desenvolvidos em amostra representativa dos adolescentes escolares da cidade de João Pessoa - PB. O único estudo que foi possível localizar, foi desenvolvido com dados do exame médico do Alistamento Militar do Exército Brasileiro para a Região Nordeste ${ }^{(8)}$.

Os dados disponíveis, até o momento, são decorrentes de levantamentos desenvolvidos em amostras não-representativas ${ }^{(8-11)}$, de representatividade regional ${ }^{(12-16)}$, e os baseados na análise dos inquéritos realizados pelo Instituto Brasileiro de Geografia e Estatística (IBGE) ${ }^{(17-19)}$ De forma em geral, estes estudos demonstram que, dependendo do lugar, do subgrupo populacional e do critério de classificação, a prevalência de sobrepeso/obesidade varia de $9 \%$ a 30\%, freqüentemente mais elevada nos rapazes em comparação às moças, e tem distribuição generalizada em relação à idade (14 a 18 anos).

Em relação à freqüência de sobrepeso/obesidade nos estratos socioeconômicos, na população jovem, têm-se observado resultados inconsistentes. Além de fatores como idade, sexo, metodologia empregada na determinação das classes socioeconômicas, critérios de classificação de sobrepeso/obesidade, e dos aspectos culturais, o estágio de desenvolvimento socioeconômico do país parece ser um dos principais fatores que interfere nessa relação(20). Nas investigações envolvendo adolescentes de países desenvolvidos, a posição socioeconômica mostra-se inversamente associada ao sobrepeso/obesidade em rapazes e moças ${ }^{(21-24)}$.

Em países em desenvolvimento, como o Brasil, apesar de divergências entre alguns estudos, observa-se maior prevalência de sobrepeso/obesidade nos rapazes de classes socioeconômicas mais elevadas ${ }^{(13,16)}$ e nas moças, dependendo da região, nas classes socioeconômicas menos abastadas ${ }^{(14)}$, ou com distribuição similar nos estratos socioeconômicos ${ }^{(13,17,25)}$.

O conhecimento sobre a prevalência de sobrepeso/obesidade e sua distribuição nos diferentes estratos socioeconômicos, permite a identificação de grupos de risco, bem como ampliar o conhecimento sobre a influência desses aspectos sobre a prevalência de sobrepeso/ obesidade, o que deverá contribuir na seleção das melhores estratégias de intervenção nesse importante problema de saúde pública. Nesse sentido, o objetivo deste estudo foi determinar a prevalência de sobrepeso/obesidade em amostra representativa dos adolescentes escolares do ensino médio, da cidade de João Pessoa - PB, e analisar sua associação com fatores demográficos e socioeconômicos.

\section{Métodos}

No desenvolvimento do estudo sobre a prevalência de sobrepeso/ obesidade e sua relação com fatores demográficos e socioeconômicos, foram utilizados os dados coletados no projeto "Comportamentos de risco à saúde em adolescentes da cidade de João Pessoa - PB", desenvolvido entre março e setembro de 2005, em uma amostra representativa de escolares do ensino médio, da cidade de João Pessoa - PB. Os protocolos de intervenção no estudo foram aprovados pelo Comitê de Ética em Pesquisa da Universidade Federal da Paraíba e acompanham normas da resolução 196/96 do Conselho Nacional de Saúde, sob pesquisa envolvendo seres humanos.

A amostragem, por conglomerado em dois estágios, foi realizada a partir dos 65 estabelecimentos de ensino médio, existentes na cidade de João Pessoa - PB. No estágio inicial, foram selecionadas de forma aleatória e proporcional, 16 escolas, sendo 10 estaduais e 6 privadas. No segundo estágio, após autorização do responsável, selecionou-se em cada escola sorteada para participar do estudo, uma quantidade de turmas, suficiente para se alcançar o número de sujeitos que garantissem a representatividade em relação à população de escolares do ensino médio, considerando a característica da escola (pública e privada) e a série de ensino (1a a 3a séries).

Na estimativa do tamanho da amostra, considerou-se uma prevalência de inatividade física de 60\%, intervalo de confiança 95\% (IC95\%), erro estimado de 3 pontos percentuais, um efeito de delineamento 2, e acréscimo de $20 \%$ para possíveis perdas/recusas. Optou-se por considerar esse desfecho na determinação do tamanho da amostra, por apresentar a maior prevalência em relação aos demais desfechos investigados no levantamento. Dados esses parâmetros, estimou-se que seria necessário coletar dados em 2.380 escolares do ensino médio. Entretanto, aplicou-se o questionário em 2.996 escolares do ensino médio. Todos os escolares da turma sorteada, que estavam na sala de aula no dia da coleta, foram considerados elegíveis para participar do estudo, mediante autorização prévia. Foram descartados os questionários respondidos pelos escolares menores de 14 anos (77 sujeitos) e maiores de 18 anos (134 sujeitos), totalizando, preliminarmente, 2.785 sujeitos.

Na seqüência, dos 2.785 escolares com idades entre 14 e 18 anos $(16,5 \pm 0,2)$, foram excluídos os que não informaram o sexo e/ou a idade ( $n=112)$, e as medidas de massa corporal e/ou estatura $(n=271)$. Em função disso, para fins deste estudo, foram considerados elegíveis para análise 2.402 escolares (1.063 rapazes e 1.339 moças).

Na coleta de dados, uma equipe composta por quatro estagiários, previamente treinados em dois estudos piloto, aplicou um questionário ${ }^{26}$ em sala de aula, que requeria 25 a 30 minutos para seu preenchimento pelos escolares. No presente estudo, foram analisadas as seguintes informações: i) demográficas (sexo e idade); ii) socioeconômicas (classe econômica, escolaridade dos pais), e medidas auto-referidas de peso $(\mathrm{kg})$ e estatura $(\mathrm{cm})$.

A idade dos escolares foi determinada com base na diferença entre a data de nascimento e a data da coleta de dados, agrupando-se as idades da seguinte forma: 14 a 14,99, e assim sucessivamente até os 18 anos de idade.

Para a definição da classe social, recorreu-se às diretrizes propostas pela Associação Brasileira de Empresas de Pesquisa (ABEP) 27, que classifica as pessoas nas classes A (alta), B, C, D e E (baixa), a partir da acumulação de bens materiais, das condições de moradia, número de empregados domésticos e o nível de escolaridade do chefe da família. Por apresentarem uma pequena quantidade de sujeitos, as categorias $A$ e E, foram reagrupadas em $A, B$ e $D, E$, permanecendo a $C$ isoladamente.

A escolaridade dos pais dos adolescentes foi considerada segundo o número de anos completos de escolaridade do chefe da família (pai/ mãe), sendo adotadas as seguintes categorias: $\leq 4$ anos, 5-8 anos, 9-11 
anos e $\geq 12$ anos. As características demográficas e socioeconômicas dos adolescentes escolares do ensino médio da cidade João Pessoa - PB, estão descritas na tabela 1.

Tabela 1. Características demográficas e socioeconômicas dos adolescentes escolares do ensino médio, João Pessoa - PB, 2005.

\begin{tabular}{|c|c|c|}
\hline Variável & $\mathrm{n}$ & $\%$ \\
\hline \multicolumn{3}{|l|}{ Sexo } \\
\hline Rapazes & 1.063 & 44,3 \\
\hline Moças & 1.339 & 55,7 \\
\hline \multicolumn{3}{|c|}{ Idade (anos) } \\
\hline 14 & 256 & 10,6 \\
\hline 15 & 529 & 22,0 \\
\hline 16 & 722 & 30,1 \\
\hline 17 & 579 & 24,1 \\
\hline 18 & 316 & 13,2 \\
\hline \multicolumn{3}{|c|}{ Classe econômica } \\
\hline $\mathrm{A}, \mathrm{B}$ (alta) & 638 & 32,4 \\
\hline C & 803 & 40,8 \\
\hline D,E (baixa) & 526 & 26,8 \\
\hline \multicolumn{3}{|c|}{ Escolaridade (anos)* } \\
\hline$\leq 4$ & 333 & 14,2 \\
\hline $5-8$ & 495 & 21,0 \\
\hline 9-11 & 777 & 33,2 \\
\hline$\geq 12$ & 742 & 31,6 \\
\hline
\end{tabular}

*Chefe da família

O índice de massa corporal (IMC= peso[kg]/estatura $\left.\left[\mathrm{m}^{2}\right]\right)$ foi obtido com base nas medidas auto-referidas (pelos adolescentes) de peso e estatura, e classificado (baixo peso/peso normal e sobrepeso/obesidade) a partir dos critérios propostos por Cole et al. ${ }^{(28)} \mathrm{O}$ IMC determinado a partir de medidas auto-referidas de peso e estatura, em substituição às medidas mensuradas, tem sido freqüentemente utilizado em levantamentos epidemiológicos que objetivam avaliar o estado nutricional de adolescentes ${ }^{(29,30)}$. Medidas auto-referidas de peso e estatura, e o IMC determinado a partir dessas medidas, mostram-se altamente correlacionadas com as medidas aferidas, e estimam, com pequena margem de erro, a prevalência de sobrepeso/obesidade em adolescentes ${ }^{(31-33)}$.

Para avaliar a associação bruta e ajustada entre variáveis demográficas (sexo e idade) e socioeconômicas (classe econômica e escolaridade dos pais) com o sobrepeso/obesidade (baixo peso/normal=0 e sobrepeso/obesidade=1), utilizou-se à medida de razão de prevalência (RP), com respectivos intervalos de confiança de 95\% (IC 95\%), a partir da regressão de Poisson. Como a literatura tem amplamente descrito que os fatores demográficos e socioeconômicos estão diferentemente associados ao excesso de peso corporal entre rapazes e moças, ambas as análises (bruta e ajustada) foram estratificadas por sexo. O teste do qui-quadrado foi utilizado para avaliar heterogeneidade e tendência linear. Todos os procedimentos de análise estatística foram realizados no programa Stata 9.0. Adotou-se um nível de significância de 5\% e todos os testes foram bicaudais.

\section{RESULTADOS}

Um em cada dez escolares do ensino médio (10\%) apresentou excesso de peso corporal (sobrepeso/obesidade). A chance de sobrepeso/obesidade foi praticamente duas vezes mais elevada $(R P=1,82$;
IC95\%=1,42-2,32) nos rapazes (13,5\%) do que nas moças $(7,4 \%)$ (dados não apresentados em tabela). A tabela 2 apresenta as prevalência e razões de prevalência de sobrepeso/obesidade brutas. A prevalência de sobrepeso/obesidade não se associou significativamente com a idade (14-18 anos) nos adolescentes de ambos os sexos. A classe econômica se associou positiva e significativamente com o sobrepeso/obesidade nos rapazes, com probabilidade 2,27 vezes maior de apresentar sobrepeso/obesidade nos que pertenciam às classes econômicas mais privilegiadas. A escolaridade dos pais não se associou ao sobrepeso/ obesidade nos escolares de ambos os sexos.

Tabela 2. Prevalência e razões de prevalência (RP) de sobrepeso/obesidade em função de variáveis demográficas ${ }^{\dagger}$ e socioeconômicas, em adolescentes escolares do ensino médio, João Pessoa - PB, 2005.

\begin{tabular}{c|c|c|c|c|c|l}
\hline \multirow{2}{*}{ Variável } & \multicolumn{2}{|c|}{ Rapazes } & \multicolumn{4}{|c}{ Moças } \\
\cline { 2 - 7 } & $\%$ & $\begin{array}{c}\text { RP bruta } \\
\text { (IC 95\%) }\end{array}$ & Valor $\mathbf{p}$ & $\%$ & $\begin{array}{c}\text { RP bruta } \\
\text { (IC 95\%) }\end{array}$ & Valor $\mathbf{p}$ \\
\hline Idade (anos) & & & $p=0,371^{*}$ & & & $p=0,109^{*}$ \\
\hline 14 & 15,6 & $1,28(0,67-2,45)$ & & 11,8 & $1,49(0,77-2,88)$ & \\
\hline 15 & 16,0 & $1,32(0,77-2,26)$ & & 8,3 & $1,05(0,56-1,96)$ & \\
\hline 16 & 14,1 & $1,16(0,69-1,97)$ & & 6,0 & $0,76(0,40-1,43)$ & \\
\hline 17 & 10,4 & $0,86(0,49-1,50)$ & & 5,5 & $0,68(0,34-1,37)$ & \\
\hline 18 & 12,1 & $1($ Referência) & & 7,9 & $1($ Referência) & \\
\hline $\begin{array}{c}\text { Classe } \\
\text { econômica }\end{array}$ & & & $p=0,004^{*}$ & & & $p=0,666^{*}$ \\
\hline A,B (alta) & 17,6 & $2,27(1,34-3,84)$ & & 7,8 & $0,91(0,47-1,32)$ & \\
\hline C & 12,2 & $1,58(0,90-2,74)$ & & 6,2 & $0,79(0,52-1,60)$ & \\
\hline D,E (baixa) & 7,7 & 1 (Referência) & & 7,2 & $1($ Referência) & \\
\hline Escolaridade \\
(anos)**
\end{tabular}

${ }^{+}$RP e IC95\% para a variável sexo (referência: moças): 1,82 (1,42-2,32) e Valor $p<0,001$ (teste para heterogeneidade);

*Teste para tendência linear;

**Escolaridade do chefe da família.

Na análise ajustada, o sexo do adolescente continuou associado ao sobrepeso/obesidade, com chance 1,87 (RP=1,87; IC95\%=1,42-2,46) mais elevada de sobrepeso/obesidade, nas moças, comparadas aos rapazes (dados não apresentados em tabela). A idade dos escolares continuou dissociada da prevalência de sobrepeso/obesidade na análise ajustada, nos adolescentes de ambos os sexos (tabela 3).

A classe econômica que na análise bruta estava positiva e significativamente associada ao sobrepeso/obesidade nos rapazes, na análise ajustada, manteve-se associada, com chance aproximadamente três vezes maior ( $R P=2,75$; IC95\%=1,56-4,82) de sobrepeso/obesidade nos que pertenciam às famílias de classes econômicas mais altas $(A, B)$, comparados aos que pertenciam às famílias menos abastadas $(D, E)$.

Nas moças, o grau de escolaridade dos pais ganhou significância estatística na análise ajustada, associando-se inversamente com o sobrepeso/obesidade. Moças cujos pais tinham 9-11 anos de escolaridade tinham menor chance de sobrepeso/obesidade ( $R P=0,42$; IC95\%=0,21-0,87), tendo como referência os pais com menor nível de escolaridade ( $\leq 4$ anos). 
Tabela 3. Análise ajustada para associação entre variáveis demográficas ${ }^{\dagger}$ e socioeconômicas com o sobrepeso/obesidade em adolescentes escolares do ensino médio, João Pessoa - PB, 2005.

\begin{tabular}{|c|c|c|}
\hline \multirow{2}{*}{ Variável } & Rapazes & Moças \\
\hline & RP ajustada (IC 95\%) & RP ajustada (IC 95\%) \\
\hline \multicolumn{3}{|l|}{ Idade (anos) } \\
\hline 14 & $1,41(0,70-2,82)$ & $1,53(0,73-3,23)$ \\
\hline 15 & $1,24(0,67-2,28)$ & $0,97(0,47-1,99)$ \\
\hline 16 & $1,26(0,71-2,25)$ & $0,65(0,31-1,38)$ \\
\hline 17 & $0,78(0,41-1,47)$ & $0,80(0,38-1,68)$ \\
\hline 18 & 1 (Referência) & 1 (Referência) \\
\hline \multicolumn{3}{|c|}{ Classe econômica } \\
\hline A,B (alta) & $2,75(1,56-4,82)$ & $1,21(0,61-2,42)$ \\
\hline C & $1,85(1,10-3,09)$ & $0,98(0,57-1,69)$ \\
\hline D,E (baixa) & 1 (Referência) & 1 (Referência) \\
\hline \multicolumn{3}{|c|}{ Escolaridade (anos) ${ }^{* *}$} \\
\hline$\leq 4$ & 1 (Referência) & 1 (Referência) \\
\hline $5-8$ & $0,80(0,43-1,50)$ & $1,01(0,55-1,88)$ \\
\hline $9-11$ & $0,61(0,33-1,10)$ & $0,42(0,21-0,87)$ \\
\hline$\geq 12$ & $0,71(0,38-1,33)$ & $0,76(0,36-1,59)$ \\
\hline
\end{tabular}

${ }^{\dagger}$ RP e IC95\% para a variável sexo (referência: moças): 1,87 (1,42-2,46), ajustada para demais variáveis (idade, classe econômica e escolaridade);

**Escolaridade do chefe da família.

\section{DISCUSSÃO}

Um em cada dez adolescentes escolares do ensino médio da cidade de João Pessoa - PB apresentou IMC acima dos valores de referência, conforme critérios propostos por Cole et al. ${ }^{(28)}$ Esses resultados são inferiores aos observados em adolescentes Norte-americanos ${ }^{(29)}$, Canadenses ${ }^{(21)}$, Franceses ${ }^{(22)}$, e Italianos ${ }^{(24)}$, cuja prevalência de sobrepeso oscilou de $22 \%$ a $29 \%$, e superiores aos descritos por Burbano et al. ${ }^{(34)}$ em adolescentes do Equador (9\%).

Por sua vez, esses resultados são similares aos encontrados em adolescentes da região Sul ${ }^{(14)}$, e Sudeste ${ }^{(15)}$ do Brasil (10\% a 12\%), e inferiores aos observados em um estudo de base populacional com adolescentes da cidade de Pelotas, região sul do Brasil $(21,8 \%)^{(13)}$. Comparados com os dados da Pesquisa sobre Padrões de Vida (PPV/IBGE)(17), a prevalência de sobrepeso/obesidade foi superior à observada na região Nordeste (8,45\%) e ligeiramente inferior a da região Sudeste do Brasil (11,53\%).

O único estudo que foi possível localizar com adolescentes escolares da cidade de João Pessoa - PB, foi desenvolvido com dados obtidos no momento da realização do exame médico do Alistamento Militar do Exército Brasileiro para a Região Nordeste. Na ocasião, verificou-se que 11,1\% desses adolescentes apresentaram quadro de sobrepeso/obesidade ${ }^{(8)}$.

A prevalência de sobrepeso/obesidade foi praticamente duas vezes mais elevada nos rapazes (13,5\%) em comparação com as moças (7,4\%). Mesmo depois de ajustado para as demais variáveis investigadas (idade, classe econômica, escolaridade dos pais), a probabilidade de apresentar sobrepeso/obesidade permaneceu mais elevada nos rapazes em comparação com as moças ( $R P=1,87 ; \mathrm{IC} 95 \%=1,42-2,46)$. Esses resultados corroboram com achados anteriores em adolescentes Europeus ${ }^{(24)}$ Norte-americanos ${ }^{(29)}$, e da região Nordeste ${ }^{(9)}$, Sudeste ${ }^{(11,15)}$ e Sul ${ }^{(14)}$ do Brasil, que também relataram maior prevalência de sobrepeso/obesidade nos rapazes. Muito embora, excepcionalmente, observa-se maior prevalência nas moças ${ }^{(35)}$ ou valores similares entre os sexos ${ }^{(13)}$.

Ao analisar os dados da PPV (1996-1997), Magalhães et al. ${ }^{(17)}$ verifi- caram diferença na prevalência de sobrepeso/obesidade entre os sexos, segundo a região que os adolescentes pertenciam. A prevalência de sobrepeso/obesidade foi mais elevada nas moças na região Nordeste (12,98\% vs 4,33\%) e nos rapazes na região Sudeste (14,64\% vs 8,48\%). É importante salientar que, desde o período de realização do inquérito, o perfil nutricional de vários países em desenvolvimento, inclusive o Brasil, tem sofrido grandes mudanças. Entre estas, uma redução na diferença da prevalência de sobrepeso/obesidade entre rapazes e moças, passando de duas a três vezes mais elevada nas moças $(1974 / 75)^{(4)}$, a mais elevada nos rapazes, mesmo em regiões menos desenvolvidas, como foi observado no presente estudo.

A idade dos escolares não se associou à freqüência de sobrepeso/obesidade, nos adolescentes de ambos os sexos, mesmo depois de ajustado para as demais variáveis (classe econômica e escolaridade dos pais). Esses resultados são consistentes com outras pesquisas ${ }^{(13,14,24)}$ que também descreveram uma dissociação entre o excesso de peso corporal e a idade dos adolescentes (14 a 18 anos).

No presente estudo, independentemente da idade do adolescente e da escolaridade dos pais, verificou-se que os rapazes que pertenciam às classes econômicas mais abastadas $(A, B)$ apresentaram maior risco de prevalência de sobrepeso/obesidade, 2,75 mais elevada, do que seus pares de classes menos favorecidas $(D, E)$. Já nas moças, a classe econômica não se associou com o sobrepeso/obesidade.

Quando se recorreu à escolaridade dos pais, como indicador de condição socioeconômica, na análise ajustada, esta se associou inversamente com o sobrepeso/obesidade nas moças, mas não nos rapazes, com redução de 58\% no risco de prevalência de sobrepeso/obesidade entre aquelas cujos pais haviam estudado 9-11 anos, tendo como referência os pais com $\leq 4$ anos de escolaridade.

As investigações, envolvendo adolescentes brasileiros, têm evidenciado uma associação positiva entre o excesso de peso corporal e a classe econômica nos rapazes, e uma dissociação nas moças ${ }^{(13,16)}$ ou uma relação inversa ${ }^{(14,36)}$. Em relação à escolaridade dos pais, esta não tem-se associado ao sobrepeso/obesidade nos adolescentes de ambos sexos ${ }^{(13)}$. Esses resultados são similares aos descritos no presente estudo: maior prevalência de sobrepeso/obesidade nos rapazes de classes econômicas mais privilegiadas e dissociação nas moças. Por outro lado, são discordantes em relação à escolaridade dos pais e sobrepeso/obesidade nas moças, que se mostraram inversamente associados: menor prevalência de sobrepeso/obesidade nas moças cujos pais apresentam maior nível de escolaridade.

Os resultados do presente estudo são discordantes daqueles encontrados em pesquisas com adolescentes de países desenvolvidos, em relação aos rapazes, os quais têm descrito uma relação inversa entre o sobrepeso/obesidade e a condição socioeconômica dos rapazes e moças ${ }^{(21-24)}$. Apesar das diferenças metodológicas (critério de classificação do IMC, indicadores de condições socioeconômicas) entre os estudos contribuírem para as divergências nos resultados encontrados, o nível de desenvolvimento socioeconômico do país parece interferir de maneira expressiva, no sentido e magnitude dessa associação(20).

A maior prevalência de rapazes com excesso de peso corporal, nas classes socioeconômicas mais privilegiadas, pode estar associada a menor preocupação com o controle do peso corporal, em detrimento ao prazer de usufruírem os benefícios decorrentes do maior acesso aos produtos eletrônicos e às redes de fast food, considerados como comportamentos socialmente aceitos, e símbolo de status nesse subgrupo populacional.

Nas moças, a relação inversa entre a condição socioeconômica e o sobrepeso/obesidade pode ser explicada pela menor aceitação social do excesso de peso, e maior valorização da forma corporal que atenda aos padrões de beleza vigentes (corpo esguio e magro) nas classes mais favorecidas. Adiciona-se a esses fatores, maior nível de conhe- 
cimento sobre as conseqüências do excesso de peso corporal, como tratar e prevenir e maior acesso aos meios que ajudam a controlar o peso corporal (alimentação saudável, prática regular de atividade física, centros de estética corporal)(21).

Mudanças no perfil nutricional, observadas nos últimos 30 anos, em países em desenvolvimento, têm levado a uma acentuação da relação inversa entre o status socioeconômico e o sobrepeso/obesidade nas mulheres e uma atenuação da relação positiva nos homens, com expectativa de assumir o padrão observado nas mulheres. Isso sugere que esses países estão caminhando para um padrão de distribuição de sobrepeso/obesidade, em função de indicadores demográficos e socioeconômicos, similar ao observado em países desenvolvidos, possivelmente, impulsionados pelo processo de globalização. Esse comportamento já vem sendo descrito em adolescentes da Região Sul do Brasil(14) e, de certa forma, no presente estudo.

Entre as principais limitações desse estudo destaca-se o fato do mesmo ser de base escolar, podendo apresentar limitações com relação à generalização dos resultados para população adolescente na sua totalidade. Entretanto, em função da elevada taxa de adolescentes matriculados nas escolas de ensino médio, em 2005, torna-se mais provável que esses resultados retratem, com condição satisfatória, a prevalência de sobrepeso/obesidade e sua distribuição nos diferentes estratos demográficos e socioeconômicos nos adolescentes da cidade de João Pessoa.

Por ser tratar de um estudo transversal, a possibilidade de se estabelecer relação causa-efeito entre a condição socioeconômica e o sobrepeso/obesidade fica comprometida. Não se pode estabelecer com clareza se o sobrepeso/obesidade tende a contribuir para menor possibilidade de ascensão social e profissional, particularmente nas

\section{REFERÊNCIAS BIBLIOGRÁFICAS}

1. Williams DP, Going SB, Lohman TG, Harsha DW, Srinivasan SR, Webber LS, et al. Body fatness and risk for elevated blood pressure, total cholesterol, and serum lipoprotein ratios in children and adolescents. Am J Public Health 1992; 82: 358-63.

2. Falkner NH, Neumark-Sztainer D, Story M, Jeffery RW, Beuhring T, Resnick MD. Social, educational, and psychological correlates of weight status in adolescents. Obes Res 2001; 9: 32-42.

3. Engeland A, Bjorge T, Sogaard AJ, Tverdal A. Body mass index in adolescence in relation to total mortality: 32-year follow-up of 227,000 Norwegian boys and girls. Am J Epidemiol 2003; 157: 517-23.

4. Wang Y, Monteiro C, Popkin BM. Trends of obesity and underweight in older children and adolescents in the United States, Brazil, China, and Russia. Am J Clin Nutr 2002; 75: 971-7.

5. Kautiaien S, Rimpelä A, Vitakt A, Virtanen SM. Secular trends in overweight and obesity among Finnish adolescents in 1977-1999. Int J Obes 2002; 26: 544-52.

6. Ogden $\mathrm{CL}$, Flegal KM, Johson CL. Prevalence and trends in overweight among US children and adolescents, 1999-2000. JAMA 2002, 288: 1728-32.

7. Veiga GV, Cunha AS, Sichieri R. Trends in overweight among adolescents living in the poorest and richest regions of Brazil. Am J Public Health 2004; 94: 1544-8.

8. Vasconcelos VL, Silva GAP. Prevalência de sobrepeso e obesidade em adolescentes masculinos, no Nordeste do Brasil, 1980-2000. Cad Saúde Pública 2003; 19: 1445-51.

9. Balaban G, Silva GAP. Prevalência de sobrepeso e obesidade em crianças e adolescentes de uma escola da rede privada de Recife. J Pediat 2001; 77: 96-100.

10. Silva GAP, Balaban, Mota MEFA. Prevalência de sobrepeso e obesidade em crianças e adolescentes de diferentes condições socioeconômicas. Rev Bras Saúde Matern Infant 2005; 5: 53-9.

11. Albano RD, Souza SB. Estado nutricional de adolescentes: "risco de sobrepeso"e "sobrepeso" em uma escola pública do município de São Paulo. Cad Saúde Pública 2001; 17: 941-7.

12. Anjos LA, Castro IRR, Engstrom EM, Azevedo AMF. Crescimento e estado nutricional em amostra probabilística de escolares no município do Rio de Janeiro, 1999. Cad Saúde Pública 2003; 19: S171-9.

13. Dutra $C L$, Araújo $C L$, Bertoldi $A D$. Prevalência de sobrepeso em adolescentes: um estudo de base populacional em uma cidade no sul do Brasil. Cad Saúde Pública 2006; 22: 151-62.

14. Farias Júnior JC, Lopes AS. Prevalência de sobrepeso em adolescentes. Revista Brasileira de Ciência e Movimento 2003; 11:77-84.

15. Ramos AMPP. Barros Filho AA. Prevalência de obesidade em adolescentes de Bragança Paulista e sua relação com a obesidade dos pais. Arq Bras Endocrinol Metab 2003; 47:663-8.

16. Campos LA, Leite AJM, Almeida PC. Nível socioeconômico e sua influência sobre a prevalência de sobrepeso e obesidade em escolares adolescentes do município de Fortaleza. Revista Nutrição de Campinas 2006; 19: 531-8.

17. Magalhães VC, Azevedo G, Mendonça S. Prevalência e fatores associados a sobrepeso e obesidade em adolescentes de 15 a 19 anos das regiões Nordeste e Sudeste do Brasil, 1996 a 1997. Cad Saúde Pública 2003; 19: 129-39.

18. Abrantes MM, Lamounier JA, Colosimo EA. Prevalência de sobrepeso e obesidade em crianças e adolescentes das regiões Sudestes e Nordeste. J Pediatr 2002; 78: 335-40. moças, e/ou se as pessoas que pertencem às classes socioeconômicas menos privilegiadas estão mais expostas aos fatores predisponentes ao aumento do peso corporal.

Outra limitação foi o uso de medidas auto-referidas de peso e estatura para estimativa da prevalência de sobrepeso/obesidade. Apesar de altamente correlacionadas com as medidas aferidas, o peso e a estatura auto-relatados pelos adolescentes tendem a ser menores do que os valores mensurados, o que pode subestimar a prevalência de sobrepeso/obesidade.

A prevalência de sobrepeso/obesidade entre os escolares de João Pessoa - PB assemelha-se ao que tem sido observado nos estados da região Sul e Sudeste do Brasil, bem como em alguns países desenvolvidos. Os rapazes, principalmente os que pertencem às classes econômicas mais abastadas e as moças cujos pais apresentaram menor nível de escolaridade, foram os subgrupos de maior exposição aos riscos decorrentes do excesso de peso corporal.

Tendo em vista o número cada vez maior de adolescentes com excesso de peso corporal, as implicações negativas à saúde, e por estar, normalmente, associado à presença de maus hábitos alimentares e ao baixo nível de atividade física, comportamentos que tendem a ser transferidos à vida adulta, é extremamente importante iniciar o mais precoce possível o desenvolvimento de ações voltadas à prevenção, controle e tratamento do excesso de peso corporal na população jovem, sendo necessário considerar a influência de forma direta e indireta dos fatores demográficos e socioeconômicos sobre esses aspectos.

Todos os autores declararam não haver qualquer potencial conflito de interesses referente a este artigo.
19. Abrantes MM, Lamounier JA, Colosimo EA, Prevalência de sobrepeso e obesidade nas regiões Nordeste e Sudeste do Brasil. Rev Assoc Med Bras 2003; 49: 162-6.

20. Martorell R, Khan LK, Hughes ML, Grummer-Strawn LM. Obesity in Latin American women and children. J Nutr 1998; 128: 1464-73.

21. Oliver $L N$, Hayes MV. Neighbourhood socio-economic status and the prevalence of overweight Canadian children and youth. Can J Public Health 2005; 96: 415-20.

22. Klein-Platat C, Wagner A, Hann MC, Arveiler D, Schlienger JL, Simon C. Prevalence and sociodemographic determinants of overweight in young French adolescents. Diabetes Metab Res Rev 2003; 19: 153-8.

23. Wang Y. Cross-national comparison of childhood obesity: the epidemic and the relationship between obesity and socioeconomic status. Int J Epidemiol 2001; 30: 1129-36.

24. Celi F, Bini V, Giorgi GD, Molinari D, Faraoni F, Stefano GD, et al. Epidemiology of overweight and obesity among school children and adolescents in three provinces of central Italy, 1993-2001: study of potential influencing variables. Eur J Clin Nutr 2003; 57: 1045-51.

25. Monteiro POA, Victora CG. Rapid growth in infancy and childhood and obesity in later life - a systematic review. Obes Rev 2005; 6: 143-54.

26. Farias Júnior JC, Pires MC, Lopes AS. Reprodutibilidade de um questionário para o levantamento de informações sobre comportamentos relacionados à saúde em adolescentes. Revista Brasileira de Ciência e Movimento 2002; 10: 43-8.

27. ABEP (Associação Brasileira de Empresas de Pesquisa). Critério de Classificação Econômica Brasil. www.abep.org.br. Disponível em: www.abep.org.br/mural/abep/cceb.htm. [Acessado em 26/11/2005].

28. Cole TJ, Bellizzi MC, Flegal KM, Dietz WH. Establishing a standard definition for child overweight and obesity worldwide: international survey. BMJ 2000; 320: 1-6.

29. Center for Disease Control and Prevention. Surveillance Summaries, May 21, 2004. MMWR 2004; 53(No.SS-2).

30. Health Education Authority (HEA). Today's young adults: 16-19 year olds look at diet, alcohol, drugs and sexual behavior. HEA: London, 1992.

31. Brener ND, McManus T, Galuska DA, Lowry R, Wechsler H. Reliability and validity of self-reported height and weight among high school students. J Adolesc Health 2003; 32: 281-7.

32. Crawley HF, Portides $\mathrm{G}$. Self-reported versus measured height, weight and body mass index amongst 16-17 year old British teenagers. Int J Obesity 1995; 19: 579-84.

33. Strauss RS. Comparison of measured and self-reported weight and height in a cross-sectional sample of young adolescents. Int J Obesity 1999; 23: 904-8.

34. Burbano JC, Fornasini M, Acosta M. Prevalencia y factores de riesgo de sobrepeso em colegialas de 12 a 19 años en una región semiurbana del Ecuador. Rev Panm Salud Publica/Pan Am J Public Health 2003; 13: 277-84

35. Santos JS, Costa MCO, Nascimento Sobrinho CL, Silva MCM, Souza KEP, Melo BO. Perfil antropométrico e consumo alimentar de adolescentes de Teixeira de Freitas - Bahia. Rev Nutr 2005; 18: 623-32.

36. Monteiro PM, Victora C, Barros F. Fatores de risco sociais, familiares e comportamentais para obesidade em adolescentes. Rev Panam Salud/Pan Am J Public Health 2004; 16: 250-8. 